\title{
How should I treat a patient with giant left main coronary artery aneurysm without associated stenosis?
}

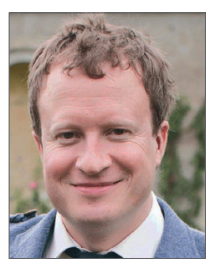

Julian O.M. Ormerod ${ }^{1}$, PhD, MRCP; Joanna Lim ${ }^{1}$, MA, MRCP;

Badri Chandrasekaran ${ }^{1}, \mathrm{MD}, \mathrm{MRCP}$; Steve Ramcharitar ${ }^{*}$, BMBCh, DPhil

1. Wiltshire Cardiac Centre, Swindon, United Kingdom

Invited experts: Scot Garg' $M B C h B, P h D, M R C P$; Ravi Singh ${ }^{2}, M B B S, M D, M R C P$; John McDonald ${ }^{2}, B S c, M B C h B, M R C P$;

Kanarath Balachandran ${ }^{2}, M B B S, M D, F R C P$; Joanna J. Wykrzykowska $, M D, P h D$

2. Department of Cardiology, East Lancashire Hospitals NHS Trust, Blackburn, United Kingdom; 3. Academic Medical Center, Amsterdam,

The Netherlands

The concluding section "How did I treat?" together with the complete references are published online at:

http://www.pcronline.com/eurointervention/92nd_issue/217

\section{CASE SUMMARY}

BACKGROUND: A 67-year-old man with a background of hypertension and hypercholesterolaemia presented to the rapid access clinic complaining of chest pain.

INVESTIGATION: CT coronary angiography showed an aneurysm of the distal left main stem. This was confirmed by coronary angiography, which revealed a $10 \mathrm{~mm}$ aneurysm of the distal left main, which involved the proximal segments of the left anterior descending and circumflex arteries. Exercise stress echocardiography showed no inducible ischaemia.

DIAGNOSIS: Giant coronary aneurysm without associated stenosis.

MANAGEMENT: Conservative.

KEYWORDS: acute coronary syndrome, atherosclerosis, coronary artery bypass grafting, giant coronary aneurysm, vasculitis

\section{PRESENTATION OF THE CASE}

The patient is a 67-year-old man who was referred to the rapid access cardiology clinic complaining of episodes of chest pain. These were atypical in nature, were not reliably related to exercise, and were occasionally occurring at rest. He had a background of hypertension and hypercholesterolaemia. There was no family history of coronary artery disease and he was a non-smoker. Exercise tolerance testing performed at the rapid access clinic was inconclusive and it was felt necessary to exclude significant coronary disease with CT coronary angiography. This showed soft plaque disease in the right coronary artery and significant calcification which warranted further investigation. A significant aneurysm of the left main bifurcation was noted (Figure 1).

The patient therefore underwent elective coronary angiography. This revealed a large aneurysm of the distal left main measuring greater than $10 \mathrm{~mm}$ and involving the bifurcation and proximal parts of the LAD and circumflex arteries (Figure 2). The vessels distal to the aneurysm were normal. There was minor atheroma throughout the right coronary artery. Magnetic resonance peripheral angiography excluded aneurysmal disease elsewhere. Connective tissue autoantibodies and syphilis serology were negative. There were no clinical features of Ehlers-Danlos syndrome.

\footnotetext{
*Corresponding author: Wiltshire Cardiac Centre, Marlborough Road, Swindon, SN3 6BB, United Kingdom. E-mail: steve.ramcharitar@chem.ox.ac.uk
} 


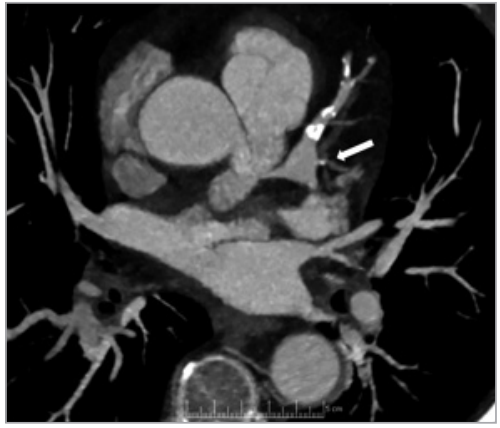

Figure 1. A computed tomography coronary angiogram showing aneurysm of the distal left main stem (arrow) with significant calcification in the left anterior descending coronary artery.

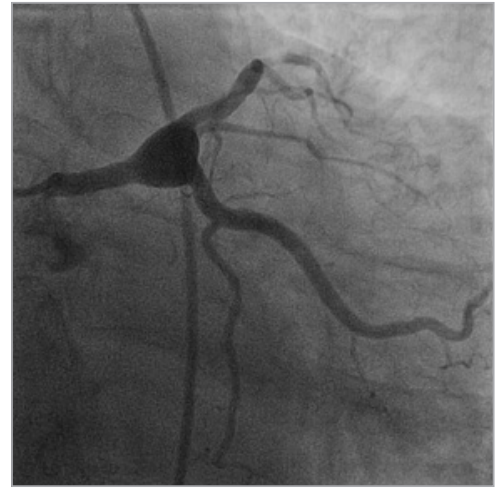

Figure 2. Coronary angiography confirming a giant aneurysm of the distal left main stem involving the bifurcation and proximal parts of the circumflex and left anterior descending coronary arteries.

\title{
How would I treat?
}

\author{
THE INVITED EXPERTS' OPINION
}

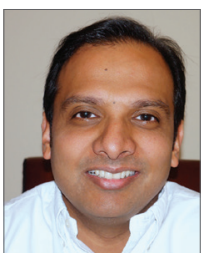

Scot Garg $2 *$, MBChB, PhD, MRCP; Ravi Singh², MBBS, MD, MRCP; John McDonald², BSc, MBChB, MRCP; Kanarath Balachandran², MBBS, MD, FRCP

2. Department of Cardiology, East Lancashire Hospitals NHS Trust, Blackburn, United Kingdom
Ormerod et al present an interesting case of a large isolated distal left main stem aneurysm. The management of this angiographic finding is challenging because its rarity ensures that data on treatment are confined to anecdotal case reports and expert consensuses. Added to this is the association between this abnormality and acute myocardial infarction and sudden cardiac death ${ }^{1}$, although this may be related to concomitant coronary stenoses rather than a direct consequence of the aneurysm. A further complexity in this case stems from the absence of any reliable history of angina pectoris, or any inducible myocardial ischaemia. Without doubt, given the potential complexities of managing this gentleman, we would discuss him at a Heart Team conference, and consider the following options.

\section{$\mathrm{PCl}$}

The patient's aneurysm extends from the left main stem into a calcified LAD, and an equally sized circumflex that could not be surrendered. We would not consider performing PCI using conventional bare metal or drug-eluting stents as this would expose the gentleman to an unacceptably high risk of stent thrombosis due to almost certain stent malapposition. A covered stent could be used to seal off the coronary aneurysm, and, whilst concerns over deliverability are not relevant in this case due to the proximal location of the aneurysm, the involvement of the bifurcation would prohibit its use. If the circumflex was small, and safe to sacrifice, a covered stent could be used. However, the long-term outcomes are unknown, and the absence of drug elution would result in a high risk of restenosis. We believe that PCI would carry a high risk of adverse events in this case.

\section{Surgical approach}

From the information provided the patient appears to have a low EuroSCORE, and therefore surgery by proximal and distal ligation of the aneurysm and bypass grafting of the distal vessels would represent a relatively low-risk option.

\section{Medical therapy PRIMUM NON NOCERE}

This patient's aneurysm does not appear to be causing any symptoms, and therefore, given the uncertainties regarding optimal management, conservative therapy is reasonable. This approach is supported by large angiographic series which have failed to demonstrate statistically significant differences in survival rates of

*Corresponding author: East Lancashire Hospitals NHS Trust, Haslingden Road, Blackburn, BB2 3HH, United Kingdom.

E-mail:scot.garg@elht.nhs.uk 
patients with coronary aneurysms treated medically or surgically when compared with controls who had similar degrees of obstructive coronary disease, but no aneurysm². Medical therapy would involve aggressive control of the patient's risk factors, together with use of antiplatelet therapy and anticoagulation. Finally, he would require regular review and serial CT scans to monitor the aneurysm's size. Any symptoms or enlargement would necessitate definitive treatment.

\section{How would I treat?}

THE INVITED EXPERT'S OPINION

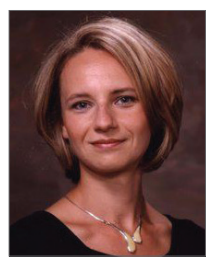

Joanna J. Wykrzykowska ${ }^{3 *}, \mathrm{MD}, \mathrm{PhD}$

3. Department of Cardiology, Academic Medical Center, Amsterdam, The Netherlands

\section{Final decision}

After deliberating, we would discuss the risks and benefits of each option with the patient. Ultimately, we feel the final choice should be between surgery and medical treatment, and the patient's views and preferences would be central to making a decision.

\section{Conflict of interest statement}

The authors have no conflicts of interest to declare.
Aneurysms of the distal left main coronary artery are extremely rare and therefore not only is the natural history poorly described but also there are no evidence-based guidelines as to the management ${ }^{2}$. If there is no associated stenosis and no demonstrable ischaemia, the two main risks associated with expectative management are: 1) distal embolisation due to sluggish flow and clotting within the aneurysm with myocardial infarction, and 2) continued aneurysm expansion and rupture/dissection. Given that the symptoms of the patient were atypical and there was no demonstrable ischaemia or contraindications to dual antiplatelet therapy or anticoagulation, perhaps medical therapy would be the best option ${ }^{3}$. The aneurysm is not due to Kawasaki disease, so perhaps warfarin is not necessary, and aspirin in combination with either prasugrel or ticagrelor could prevent clotting and embolisation risk. Further intravascular imaging with IVUS could perhaps shed light on the presence of thrombus or its absence. In the absence of connective tissue disorders and inflammatory disorders, the risk of rapid aneurysm expansion is lower and perhaps one could follow the size of the aneurysm with a repeat CT sc an over six to 12 months. Only with evidence of rapid enlargement would an intervention be indicated. In the latter case, surgical management in combination with interventional management would probably address the two risks of dissection and embolisation ${ }^{4}$. Distal left main is usually not so easily surgically accessible to surgeons for over-sewing. I would propose asking the surgeons to place arterial bypass on the LAD (LIMA to $\mathrm{LAD}$, possibly minimally invasively). Subsequently, one could exclude the aneurysm with a covered stent from the left main into the RCX (jailing the bypassed LAD). The whole procedure could possibly be performed in one session in a hybrid operating room 5 .

\section{Conflict of interest statement}

The author has no conflicts of interest to declare. 


\section{How did I treat?}

\section{ACTUAL TREATMENT AND MANAGEMENT OF THE CASE}

\section{Why we did not treat}

Coronary artery ectasia is not in itself rare, with some degree of ectasia found in between 1:12 and 1:30 patients at angiography. The prevalence in the general population is lower, between 1:70 and 1:500 in autopsy series ${ }^{2,6-8}$. It is defined as an arterial diameter 1.5 times greater than that of the adjacent vessel. It is most commonly found in association with atherosclerosis, but may be secondary to Kawasaki disease, connective tissue disease, various vasculitides, mycotic vascular damage or cocaine use. The most common histologic feature is destruction of the vessel media. The putative mechanisms include ulceration with positive remodelling, chronic nitric oxide-mediated overstimulation, and direct damage by inflammatory mediators. Coronary artery ectasia in the absence of significant occlusive disease is substantially less common, and poses a therapeutic challenge. Arterial diameters of greater than $10 \mathrm{~mm}$, such as in our patient, are very rare. We discussed the patient in our medical/surgical multidisciplinary team meeting. We had three main concerns in mind when deciding upon our management strategy: the risk of thrombosis, that of further chest pain (or ACS), and the risk of rupture.

\section{Risk of thrombosis}

The risk of thrombosis is generally considered to be low, but thrombus certainly may be found in giant aneurysms, and there is a risk of embolisation into the distal vesse ${ }^{9}$. Anticoagulation with warfarin has been used on the basis of the observed sluggish flow within the ectatic segment, but is no longer considered optimal ${ }^{10}$. There are no randomised trials to guide management but anecdotal reports support the use of antiplatelet therapy ${ }^{11}$. This is indicated in those patients with plaque disease who present with acute coronary syndrome, or post $\mathrm{CABG}$, but it is untested in ectasia without plaque disease. We opted to treat the patient with dual antiplatelet therapy.

\section{Risk of an ischaemic event}

Ectatic arterial segments display reduced flow: this can lead to both reduced distal flow and microembolisation, which may be experienced by the patient as chest pain. Exercise stress echocardiography in this patient was reassuring, showing normal left ventricular systolic function at rest with no regional wall abnormalities and no evidence of inducible ischaemia at peak exercise. This conclusively excluded any functional obstruction within or preceding the aneurysmal segment.

\section{Risk of rupture}

According to Laplace's equation, tension in the aneurysm wall at a given pressure increases proportionately with the radius. Larger aneurysms are more likely to rupture, but, in contrast to our extensive knowledge of abdominal aortic aneurysm, data as to the rate at which this might occur in coronary aneurysms are lacking. Rupture was therefore a significant concern, something which has been reported in the literature ${ }^{12}$. Coronary artery ectasia is more common in those patients who have aneurysms elsewhere ${ }^{13,14}$. These are commonly found in the aorta or iliac, popliteal, or pulmonary arteries, and occasionally in large veins. Such patients may suffer from a generalised defect of the vascular wall and so this risk of rupture may be higher. Our patient underwent MRI arteriography, which excluded disease elsewhere. This may make the risk of rupture lower, but this assumption is not evidence-based.

Monitoring vessel wall thickness is possible with intravascular ultrasound (IVUS), but the amount of ionising radiation required by regular coronary angiography, as well as the risk of injuring the abnormal vessel with the relatively stiff IVUS probe, was considered unacceptable. Improvements in non-invasive imaging technology may make such an approach feasible in the future.

\section{Treatment options}

We considered several treatment strategies in consultation with the cardiothoracic surgery team. The first option would be a left main bypass, with grafts to the proximal LAD and circumflex arteries. The ectatic segment would need to be tied off, as otherwise the grafts would be at risk of occlusion. A complex percutaneous option could be conceived. This would probably include some form of covered stent technique, with refashioning of the left main stem bifurcation. The final option would be a conservative approach.

The natural history of coronary artery aneurysm is not well studied, but it appears that the majority of the risk of such lesions is 
due to associated atherosclerosis, rather than the dilatation itself ${ }^{2,10}$. With this in mind, in this patient without significant atheroma, we opted for a conservative strategy.

\section{Conclusion}

We recommended conservative management with close followup. The patient was in agreement. He was not at all keen to go for surgery and this was a factor in our decision making. Repeat CT coronary angiography at six and 12 months was unchanged. We continue to have an open mind, but would certainly re-explore the option of surgery should annual CT coronary angiography show significant changes in architecture or size, or should the patient develop intractable symptoms. This case illustrates the difficulty of managing risk in unusual circumstances, where evidence is scant. Sometimes the most challenging decision is to do nothing.

\section{Conflict of interest statement}

The authors have no conflicts of interest to declare.

\section{References}

1. Baman TS, Cole JH, Devireddy CM, Sperling LS. Risk factors and outcomes in patients with coronary artery aneurysms. Am J Cardiol. 2004;93:1549-51.

2. Swaye PS, Fisher LD, Litwin P, Vignola PA, Judkins MP, Kemp HG, Mudd JG, Gosselin AJ. Aneurysmal coronary artery disease. Circulation. 1983;67:134-8.

3. Moreno N, Gonçalo L, Castro A, Andrade A, Rodrigues JA, Azevedo J, Ribeiro VG, Pinto P. Left main coronary artery aneurysm. Rev Port Cardiol. 2011;30:65-72.

4. Bairaktaris A, Schenk S, Grimmig O, Koerfer R. Surgical correction of a giant aneurysm of the left main coronary artery. Eur J Cardiothorac Surg. 2008;34:668.
5. Reicher B, Poston R, Mehta MR, Joshi A, Odonkor P, Kon Z, Reyes PA, Zimrin DA. Simultaneous "hybrid" percutaneous coronary intervention and minimally invasive bypass grafting: feasibility, safety and clinical outcomes. Am Heart J. 2008;155:661-7.

6. Hartnell GG, Parnell BM, Pridie RB. Coronary artery ectasia, its prevalence and clinical significance in 4993 patients. Br Heart J. 1985;54:392-5.

7. Markis JE, Joffe CD, Cohn PF, Feen DJ, Herman MV, Gorlin R. Clinical significance of coronary arterial ectasia. Am J Cardiol. 1976;37:217-22.

8. Oliveros RA, Falsetti HL, Carroll RJ, Heinle RA, Ryan GF. Atherosclerotic coronary artery aneurysm. Report of five cases and review of literature. Arch Intern Med. 1974;134:1072-6.

9. Wilkinson JS, Schulte P, Moustapha A, Dehghani P. Use of cardiac computed tomography in the management of symptomatic coronary aneurysm: a case study and literature review. Exp Clin Cardiol. 2012;17:69-73.

10. Demopoulos VP, Olympios CD, Fakiolas CN, Pissimissis EG, Economides NM, Adamopoulou E, Foussas SG, Cokkinos DV. The natural history of aneurysmal coronary artery disease. Heart. 1997; 78:136-41.

11. Mavrogeni S. Coronary artery ectasia: from diagnosis to treatment. Hellenic J Cardiol. 2010;51:158-63.

12. Daneshvar DA, Czak S, Patil A, Wasserman PG, Coplan NL, Garratt KN. Spontaneous rupture of a left main coronary artery aneurysm. Circ Cardiovasc Interv. 2012;5:e63-5.

13. Stajduhar KC, Laird JR, Rogan KM, Wortham DC. Coronary arterial ectasia: increased prevalence in patients with abdominal aortic aneurysm as compared to occlusive atherosclerotic peripheral vascular disease. Am Heart J. 1993;125:86-92.

14. Daoud AS, Pankin D, Tulgan H, Florentin RA. Aneurysms of the coronary artery. Report of ten cases and review of literature. Am J Cardiol. 1963;11:228-37. 